\title{
Concomitant Occurrence of Segmental Neurofibromatosis and Lung Adenocarcinoma
}

\author{
Ryoko Morita, Naoki Oiso", Akira Kawada \\ Department of Dermatology, Faculty of Medicine, Kinki University, Osaka, Japan. \\ Email: "naoiso@med.kindai.ac.jp
}

Received September $27^{\text {th }}, 2012$; revised October $25^{\text {th }}, 2012$; accepted November $6^{\text {th }}, 2012$

\begin{abstract}
Neurofibromatosis type 1 (NF1) caused by a loss-of functional mutation in NF1 encoding neurofibromin is an autosomal dominant disorder characterized by café-au-lait spots, neurofibromas, intertriginous freckles, and Lisch nodules. Segmental neurofibromatosis (SN) represents a postzygotic mutation and loss of heterozygosity in neurofibromin. SN occurring in the elder persons may be associated with internal malignant tumors. Here, we reported a case of 58-year-old woman with concomitant occurrence of SN and lung adenocarcinoma. The onset of SN in aged persons would be a sign of concomitant occurrence of internal malignant tumors.
\end{abstract}

Keywords: Segmental Neurofibromatosis; Lung Adenocarcinoma; Internal Malignant Tumors

\section{Introduction}

Neurofibromatosis type 1 (NF1) caused by a loss-of functional mutation in NF1 encoding neurofibromin is an autosomal dominant disorder characterized by café-aulait spots, neurofibromas, intertriginous freckles, and Lisch nodules. Segmental neurofibromatosis (SN) essentially represents a postzygotic mutation and loss of heterozygosity in neurofibromin. The age of onset of $\mathrm{SN}$ is bimodal, at $10-30$ and $50-70$ years. SN in the elders may be associated with internal malignant tumors, including bronchoalveolar lung carcinoma, colon adenocarcimona, gastric adenocarcinoma, and Hodgkin lymphoma [1-4]. We describe concomitant occurrence of SN and lung adenocarcinoma in a 58-year-old woman.

\section{Case Report}

A 58-year-old woman, a life-long nonsmoker, was referred to us with asymptomatic soft papules, $3-7 \mathrm{~mm}$ in size, on the left back (Figure 1). The papules first began to appear 2 years before and were located in the same segmental region. Café-au-lait spots, intertriginous freckling and musculoskeletal abnormalities were not present. The family had no history of pigment disorders or parental consanguinity. One year earlier, the patient had been diagnosed with lung adenocarcinoma, so the papules were present one year before the diagnosis. The skin tumors were surgically excised. The histopathological specimens from the papules showed non-encapsulated, faintly eosinophilic tumors in the dermis. The tumors were com- posed of thin spindle cells with elongated and wavy nuclei. The papules were diagnosed as SN.

\section{Discussion}

Neurofibromin is expressed at low levels in all cells, but at higher levels in the nervous system. It functions as a negative regulator of the Ras/mitogen-activated protein kinase (MAPK) signaling pathway via suppression of active Ras. As the Ras/MAPK cascade is crucial for controlling cellular growth and differentiation, a lack of functional neurofibromin results in the constitutive activation of this central signaling pathway and unregulated cell growth [5]. Somatic NF1 gene mutations have been identified not only in SN but also in various neoplasms derived from internal organs.

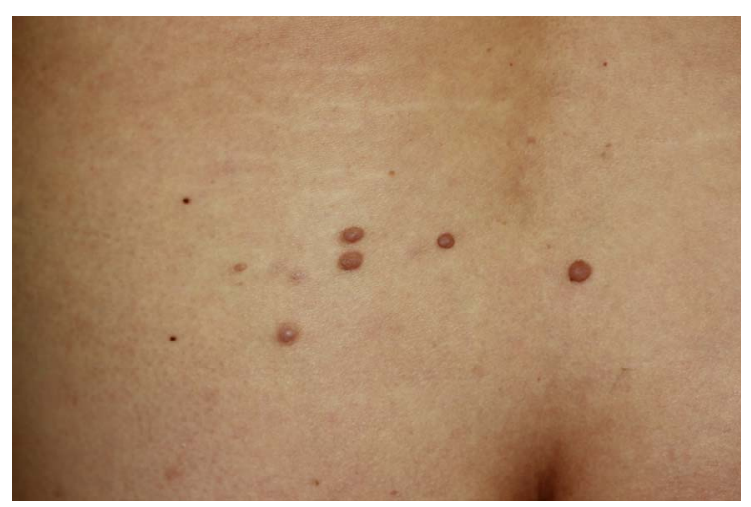

Figure 1. A clinical picture of asymptomatic soft papules, 3 $7 \mathrm{~mm}$ in size, on the left back. 
The tendency to develop SN would be predetermined during embryogenesis with the formation of clonal predisposed cells, and exposure to an appropriate trigger would result in the development of SN. Similarly, the predisposed condition toward carcinogenesis in the lung would be formed with the formation of clonal predisposed cells, and concomitant exposure to an appropriate trigger would result in the development of lung adenocarcimoma.

Bourdeaut et al. reported an infant with activated mosaicism for oncogenic KRAS mutation with occurrence of epidermal nevi, rhabdomyosarcoma, and polycystic kidneys [6]. The persistent activated condition of the Ras/ MAPK signaling pathway in the skin and other organs could be caused by either somatic gain-of-function mutations in the oncogenic genes or somatic loss-of-function mutations in the tumor suppressor genes. We surmise that constitutive activated condition of the Ras/MAPK signaling pathway in the skin and other organs is associated with the concomitant development of the proliferative skin diseases and tumorigenetic disorders in the other organs.

Further accumulation of cases with concomitant SN and malignant neoplasm is indispensable, because carcinomas such as lung adenocarcinoma are not rare. Molecular study with both tissues would reveal whether the concomitant occurrence is caused by the simultaneous damage of the same cascade in both skin and other organs or by the mere fortuitous coincidental situation.

In summary, we report a case of simultaneous presence of SN and lung adenocarcinoma and speculate the constitutive activated Ras/MAPK signaling pathway. The onset of SN in the aged persons would be a sign of con- comitant occurrence of internal malignant tumors.

\section{REFERENCES}

[1] S. E. Kim, E. P. Heo, T. J. Yoon and T. H. Kim, "Segmentally Distributed Neurofibromatosis Associated with Adenocarcinoma of the Colon," The Journal of Dermatology, Vol. 29, No. 6, 2002, pp. 350-353.

[2] B. Yalçin, G. G. Toy, E. Tamer, P. Oztaş, D. Koç, B. Dikicier and N. Alli, "Increased Expression of Segmental Neurofibromatosis with Bronchoalveolar Lung Carcinoma," Dermatology, Vol. 209, No. 4, 2004, p. 342. doi: $10.1159 / 000080862$

[3] A. Kajimoto, N. Oiso, K. Fukai and M. Ishii, "Bilateral Segmental Neurofibromatosis with Gastric Carcinoma," Clinical and Experimental Dermatology, Vol. 32, No. 1, 2007, pp. 43-44.

[4] J. D. Dang and P. R. Cohen, "Segmental Neurofibromatosis of the Distal Arm in a Man Who Developed Hodgkin Lymphoma," International Journal of Dermatology, Vol. 48, No. 10, 2009, pp. 1105-1109. doi:10.1111/j.1365-4632.2009.04132.x

[5] S. L. van Spyk, N. Thomas, D. N. Cooper and M. Upadhyaya, "Neurofibromatosis Type 1-Associated Tumours: Their Somatic Mutational Spectrum and Pathogenesis," Hum Genomics, No. 5, Vol. 6, 2011, pp. 623-690.

[6] F. Bourdeaut, A. Hérault, D. Gentien, G. Pierron, S. Ballet, S. Reynaud, R. Paris, G. Schleiermacher, C. Baumann, P. Philippe-Chomette, M. Gauthier-Villars, M. Peuchmaur, F. Radvanyi and O. Delattre, "Mosaicism for Oncogenic G12D KRAS Mutation Associated with Epidermal Nevus, Polycystic Kidneys and Rhabdomyosarcoma," Journal of Medical Genetics, Vol. 47, No. 12, 2010, pp. 859-862. doi:10.1136/jmg.2009.075374 